\title{
Finite-dimensional dynamical system modeling thermal instabilities
}

\author{
Michael L. Frankel ${ }^{\mathrm{a}}$, Gregor Kovačič ${ }^{\mathrm{b}}$, Victor Roytburd ${ }^{\mathrm{b}, *}$, Ilya Timofeyev $^{\mathrm{b}}$ \\ a Department of Mathematical Sciences, Indiana University-Purdue University at Indianapolis, Indianapolis, IN 46202-3216, USA \\ ${ }^{\mathrm{b}}$ Department of Mathematical Sciences, Rensselaer Polytechnic Institute, Troy, NY 12180-3590, USA \\ Received 22 December 1997; received in revised form 19 March 1999; accepted 23 July 1999 \\ Communicated by C.K.R.T. Jones
}

\begin{abstract}
We describe a three-dimensional dynamical system, which is obtained as a pseudo-spectral approximation to a free boundary problem modeling solid combustion and rapid solidification, and is capable of generating its major dynamical patterns. These patterns include a Hopf bifurcation followed by a sequence of secondary period doubling and a transition to chaos, reverse sequences, and sequences followed by Shilnikov type trajectories. A computer-assisted bifurcation analysis uncovers some novel mechanisms of stability exchange. The most striking of them is an infinite period bifurcation which resembles the classical Shilnikov bifurcation, but instead of a funnel-shaped spiral along which the period is continually increasing, the continuation produced a series of isolas. Each isola is a closed branch of solutions of roughly the same period, and with the same number of oscillations. The isolas corresponding to consecutive numbers of low amplitude oscillations about the equilibrium are adjacent to each other, and appear to accumulate on a saddle-focus homoclinic connection of Shilnikov type. (C)2000 Elsevier Science B.V. All rights reserved.
\end{abstract}

Keywords: Hopf bifurcation; Shilnikov bifurcation; Free boundary problems

\section{Introduction}

In this paper we introduce and study a three-dimensional system of ordinary differential equations (see (4.6)-(4.8)) capable of generating a variety of non-trivial dynamics. This system is one of a handful of examples of differential equations that provide "real life", working models for constructions of the dynamical systems theory. The system arises as a pseudo-spectral approximation of a free boundary problem for the heat equation, which describes condensed phase combustion and some exothermic non-equilibrium phase transitions. In a wide range of parameters the three-dimensional dynamical system gives a qualitatively accurate approximation of the free boundary problem and reproduces its major dynamical patterns.

Evolution of exothermic interfaces generates a remarkable variety of spatio-temporal patterns. These patterns arise when, in certain ranges of parameters, uniformly traveling modes of propagation become unstable, and undergo

\footnotetext{
* Corresponding author.

E-mail address: roytbv@ rpi.edu (V. Roytburd).
} 
a transition to self-oscillatory regimes. Such transitions have been observed in experiments for both the condensed phase and the premixed gaseous combustion [20,22]. Similar instabilities have been observed in the process of rapid solidification of thin films initiated by a laser beam (see [23]), and laser-induced evaporation of solid materials [15]. A variety of complex (including chaotic) auto-oscillatory regimes have also been found in numerical simulations of mathematical models of different degrees of complexity. In particular, in our recent work [6-10] we undertook a systematic investigation of a "one-phase" free boundary problem.

As was demonstrated in [6], dynamics of the free boundary (in one spatial dimension the free boundary is just a point moving along the $x$-axis) includes such different scenarios as a uniform motion, a Hopf bifurcation and persistent simple periodic oscillations for any overcritical value of the control parameter, a Hopf bifurcation followed by a sequence of secondary period doubling bifurcations resulting in a transition to chaos, reverse sequences, sequences followed by Shilnikov type trajectories, infinite period bifurcations, etc. It is rather remarkable that such a variety of dynamical patterns is exhibited by a relatively simple problem, which consists of the 1-D heat equation and (free) boundary conditions. This dynamical abundance is predicated on the subtle interaction between the heat release at the free boundary (governed by the boundary kinetics) and the thermal diffusion in the medium.

Results of numerical experiments with the free boundary model demonstrate surprisingly clear-cut patterns, some of which are well known and have been studied extensively for the finite-dimensional dynamical systems. It leads quite naturally to the conjecture that dynamics generated by the free boundary problem may be essentially finite-dimensional. The low-dimensional qualitative approximation of the free boundary problem that is introduced in the present paper gives substantial, although indirect, evidence in support of this conjecture.

For the derivation of the finite-dimensional model we employ a pseudo-spectral method with the basis formed by the Laguerre polynomials. This is a very natural basis for the problem, in view of the uniform exponential decay of solutions along the spatial variable. The issue of a correct choice of the discrete approximation method turns out to be extremely important. Perhaps partially due to the success of the Lorenz model, the Galerkin method has been used almost exclusively in the attempts to derive finite-dimensional approximations of systems governed by PDEs. In our case, however, no reasonable imitation of the original problem, as it seems, could be achieved based on the Galerkin approximation with a low number of modes. One plausible explanation could be that the dynamical patterns described above occur for highly overcritical values of the bifurcation parameter and far away (in any reasonable norm) from the basic solution, so that any sense of the orthogonality associated with the linearization is completely lost.

Discrete approximation based on the collocation method appears much more successful. The three-dimensional dynamical system introduced below generates a remarkably accurate qualitative imitation of the original free boundary problem, especially taking into account that only three collocation points have been used. In fact, certain subtleties in the dynamics of the original problem that we had not noticed in earlier simulations on the free boundary model were discovered via the three-dimensional model. The dynamics persists with the increase of number of collocation points, while spectra of the corresponding linearizations approach the linear spectrum of the prototype PDE problem.

The rest of the paper is organized as follows. In Sections 2 and 3, for the reader's convenience, we describe the underlying free boundary model, summarize its analytical properties and present certain relevant features of its dynamical behavior. In Section 4 through the pseudo-spectral approximation, we derive a three-dimensional system of ODEs that is the main subject of the paper. This section also includes a comparison of spectral properties of successive approximations which are based on increasing number of collocation points.

Section 5 is central for the paper: it gives a detailed description of dynamics exhibited by the system of ODEs. We present results of numerical simulations that demonstrate a rich variety of dynamical scenarios corresponding to different kinetic functions inherited from the free boundary problem. Comparison with observations of dynamics generated by the original problem shows a uniform qualitative agreement between them, practically throughout the 
entire parameter space. The bifurcation structure of the problem is investigated with the aid of the AUTO software package [4]. We describe novel (and not yet completely understood) mechanisms of exchange of stability that are suggested by the bifurcation diagrams. In particular, we would like to single out an infinite period bifurcation which superficially appears to be similar to the classical Shilnikov bifurcation [14]. Periodic solutions for this type of bifurcation have the following structure: they contain a longer duration, high amplitude excursion from the equilibrium which is followed by a series of high frequency, low amplitude oscillations about the equilibrium. For the classical Shilnikov case, the bifurcation curve is oscillatory, with the magnitude of oscillations gradually decaying (a "snake"); the period of periodic solutions is continually increasing and approaching infinity along the bifurcation curve. In contrast to the classical Shilnikov bifurcation, in our case the continuation produced an isola, a closed branch of solutions of roughly the same period, and with the same number of oscillations. The isolas corresponding to consecutive numbers of low amplitude oscillations are adjacent to each other. Periodic solutions bifurcate along the envelope of the isolas, which appears to be a parabola. In this sense, the exchange of stability exhibited by this infinite period bifurcation is analogous to the "soft" loss of stability for a supercritical Hopf bifurcation. A derivation of the $3 \times 3$ system and some results of the paper were announced in [11].

\section{Motivation of the free boundary model}

In this section we sketch the derivation of the free boundary model which is at the heart of the present investigation. We consider condensed phase combustion. For this type of combustion a solid fuel mixture is transformed directly into a solid product. In addition to its theoretical interest, gasless combustion currently finds technological applications as a method of synthesizing certain ceramics and metallic alloys [21]. The most primitive model of gasless combustion involves a system of differential equations for the temperature $u$ and the concentration of the fuel $C$ (see [22]). In the one-dimensional formulation it takes the form:

$$
\begin{aligned}
& u_{t}=\left(\kappa u_{x}\right)_{x}+q W(C, u), \\
& C_{t}=-W(C, u),
\end{aligned}
$$

where $\kappa$ is the thermal diffusivity, $W$ is the chemical reaction rate, and $q$ is the heat release.

For physically relevant values of parameters, the system is characterized by the strong temperature sensitivity of the rate and by rather sharply defined regions of dramatic change in the field variables that are usually associated with propagating fronts. This suggests an alternative to the models with distributed kinetics which is provided by those with concentrated kinetics (so-called flame sheet approximation, see [25]). The distributed reaction rate in (2.1) and (2.2) is replaced by the $\delta$-function,

$$
W=w(u) \delta(x-s(t)),
$$

supported at the interface $x=s(t)$ between the fresh $(C=1)$ and burnt $(C=0)$ material (see [19]). In the case of gaseous combustion when the distributed kinetics is of the Arrhenius type, the $\delta$-function model is the rational asymptotic limit of the distributed kinetics model in the large activation energy limit. In this case the strength of the $\delta$-function $w(u)$ is determined through an asymptotic analysis by matching relevant inner and outer solutions. Of course, all the intricacies of the behavior in the reaction zone are lost in this approximation.

The system (2.1) and (2.2) with the $\delta$-function source is understood in the sense of distributions. This leads to the system of two heat equations coupled at the interface:

$$
\begin{aligned}
& u_{t}^{-}=\left(\kappa u_{x}^{-}\right)_{x}, \quad u_{t}^{+}=\left(\kappa u_{x}^{+}\right)_{x},\left.\quad u^{-}\right|_{x=s(t)}=\left.u^{+}\right|_{x=s(t)}, \\
& \left(\kappa u_{x}^{+}-\kappa u_{x}^{-}\right)_{x=s(t)}=-w(u)_{x=s(t)}, \quad \frac{\mathrm{d} s}{\mathrm{~d} t}=-\left.w(u)\right|_{x=s(t),}
\end{aligned}
$$


where

$$
u^{-}(x, t)=u(x, t) \quad \text { for } x<s(t), \quad u^{+}(x, t)=u(x, t) \text { for } x>s(t) .
$$

This is the free interface two-phase problem of condensed phase combustion. The heat conductivity coefficient $\kappa$ is usually considered to be a constant. But, in principle, the heat conductivities of the fuel and of the product may be drastically different. For example, if the product is a foam-like substance then $\kappa_{\text {product }} \ll \kappa_{\text {fuel }}$. By setting $\kappa_{\text {product }}=0$ in the equation and the boundary condition for $u^{+}$in (2.4), we arrive at the following one-phase model problem for $u=u^{+}$:

$$
u_{t}=u_{x x}, \quad-\infty<x<s(t)
$$

with the Stefan type conditions at the free boundary,

$$
\left.u\right|_{x=s(t)}=g(\dot{s}(t)), \quad \partial u /\left.\partial x\right|_{x=s(t)}=-\dot{s}(t),
$$

where $s(t)$ is the position of the free boundary and $\dot{s}(t)$ is its velocity. The concrete form of the dimensionless version of the nonlinearity $g$,

$$
g(\dot{s})=1+v K(-\dot{s}), \quad \text { where } K(1)=0, K^{\prime}(1)=1,
$$

is introduced in Section 3. A very similar one-phase model was introduced earlier in the context of laser induced evaporation from the surface of metals [15] or for impurity controlled solidification [18].

We note that in the context of condensed phase combustion, the kinetic boundary condition in (2.6) expresses the dependence of the propagation velocity on the temperature of the flame front. In the context of solidification of overcooled liquids (see, e.g., [5]) or the amorphous to crystalline transition (see [18,23]) the condition corresponds to the interface attachment kinetics, which are determined by various microscopic mechanisms of incorporating the product phase into the crystalline lattice at the interface.

\section{Dynamics of the free boundary model}

With the normalizations stated in (2.7), it is easy to see that the free boundary model (2.5) and (2.6) supports the traveling wave solution,

$$
u_{b}(x, t)=\mathrm{e}^{x+t} \text { for } x \leq-t ; \quad s_{b}(t)=-t .
$$

that moves with velocity $V=\dot{s}(t)=-1$. It is the only traveling wave solution, provided the kinetic function $g$ is monotone.

The rate of change of $g(V)$ at $V=-1$ is a very important characteristic of the chemical reaction. For the asymptotics employed in [19], the inverse to the kinetic function $g$ is given by $V=-\exp [\alpha(u-1)]$ or

$$
u=g(V)=1+\frac{1}{\alpha} \ln (-V),
$$

where $\alpha$ (the Zeldovich number) is proportional to the dimensionless activation energy. As a parameter responsible for the sensitivity of the reaction with respect to temperature variations, we utilize $v=1 / \alpha$. We will use the kinetic function in the form:

$$
g(V)=1+v K(-V), \quad \text { where } K(1)=0, K^{\prime}(1)=1 .
$$

The free boundary problem in (2.5) and (2.6) is well posed for a wide class of kinetic functions $g=1+v K$. It was proved in $[8,10]$ that if the inverse function $g^{-1}$ is monotone and bounded from $0, V=g^{-1}(u)<-v_{0}$ for 
$u \geq 0$, and its growth for large $u$ is bounded by a "slightly" sublinear function, $\sim C u^{1 /(1+\gamma)}$, where $\gamma>0.132$ is a constant, then a classical solution exists for any once differentiable initial data that are consistent with the boundary conditions at time 0 . The solution exists for all time, and the corresponding free boundary velocity is bounded. Note that the important case of Arrhenius kinetics is included, since in that case $g^{-1}(u)$ is bounded for large $u$. The well-posedness result is obtained through a rather clever use of parabolic potentials. It appears that the excess sublinearity $\gamma>0$ is due more to the method of [10] than the nature of the problem. At least in the case of the two-phase version of the problem $\gamma$ is equal to 0 [12]. We should note an earlier work by Visintin [24] where the existence of weak solutions had been established for a related two-phase problem in a bounded interval.

Some indications of the complex dynamics generated by the model can be obtained even at the level of linear analysis (see [9] for detail). Consider small perturbations of the basic solution (3.1) which in the front attached coordinate system take the form $u=\epsilon \mathrm{e}^{\lambda t} f(x), \dot{s}=\epsilon \mathrm{e}^{\lambda t}$ and drop all the terms of order higher than $\epsilon$. To satisfy the linearized differential equation and boundary conditions, one is led to the following dispersion relation:

$$
v^{2} \lambda^{2}+(3 v-1) \lambda+v=0
$$

which produces two eigenvalues

$$
\lambda=\frac{1-3 v \pm(1-v) \sqrt{1-4 v}}{2 v^{2}} .
$$

The real part of the eigenvalues changes sign from negative to positive as $v$ decreases through the threshold $v_{\mathrm{cr}}=$ $1 / 3$; the eigenvalues cross the imaginary axis transversally. In addition to the two points of discrete spectrum, the linearized operator has the continuous spectrum filling the set $(-\infty,-1 / 4]$. Thus, one can expect a Hopf bifurcation at $v=v_{\mathrm{cr}}$, which is indeed the case as was proved in [9].

The situation, however, is much more interesting and complicated than just a Hopf bifurcation, if a physically relevant case of a parametric family of kinetic functions is considered. Concerning the choice of the kinetic function we remark that this issue is far from settled either theoretically or experimentally. For example, for solid combustion the widely used Arrhenius kinetics has not been obtained from an analysis of molecular collisions in the spirit of the kinetic theory of gases, but has been, to a degree, "transplanted" from gas combustion. There are several types of functions that were suggested for a more realistic description of kinetics in specific chemical and physical settings. On the other hand, the exact form of the interface attachment kinetics for solidification fronts may vary, and to the best of our knowledge, has not been reliably established. For example, Brailovsky and Sivashinsky [1] suggested to employ a family of kinetic functions with the extra parameter related to a temperature ratio that is characteristic for the uniform propagation of the reaction.

In our work [6] we also used another one-parameter family $K_{p}(V)$ of kinetic functions that has a simple, power behavior for large $|V|$ and similarly to (3.2) has a singularity at $V=0$ :

$$
K_{p, q}(-V)=\frac{V^{p}-V^{-q}}{p+q}, V>0 .
$$

The power kinetics behaves very similarly to the Arrhenius kinetics (see Fig. 1 of [6]). It also appears that dynamical scenarios are, in some sense, "homeomorphic" to each other for different $q$ 's. Everywhere in this paper we keep $q$ fixed at $q=1$. Note that the extra parameter $p$ is nonlinear in the sense that it does not make any contribution to the linearization.

Results of numerical simulations of [6] are summarized next. We note that dynamical scenarios are identical, no matter whether kinetics $K_{p}$ or the kinetics from [1] are used. It appears that what does matter is the presence of two bifurcation parameters, ( $v, p$ in our case); only the $K_{p}$ kinetics will be discussed below. We also note that the non-trivial dynamics for numerical simulations with distributed kinetics [2], also require an extra parameter, which 
in this case is related to melting of the solid before burning. In the figures below, we illustrate dynamics by graphs of time evolution of the velocity perturbation $v(t)$ over the basic velocity, $v=V-(-1)$. To give a better idea about the phase space behavior of the solution, we also plot $v(t)$ against the value of the solution at a fixed distance ahead of the front, $u(-1, t)$. The latter picture is a two-dimensional projection of the infinitely dimensional phase space $u(x, \cdot), v(\cdot)$.

All the regimes discussed below are initiated by a small deviation from the basic profile and velocity. The time histories and phase diagrams are shown only for sufficiently large times when the transient behavior is eliminated. For $v>v_{\text {cr }}=1 / 3$, the basic traveling wave is stable (the local exponential stability was proved in [9]). As $v$ drops below $v_{\mathrm{cr}}$ there are two principal types of stability exchange, depending on the value of the second bifurcation parameter, $p$. For the first type, exchange of stability resembles a Shilnikov bifurcation (see e.g., [14]) and for the second type, exchange of stability starts as a Hopf bifurcation (which is followed by a period doubling cascade and further, more complicated evolution).

The Shilnikov type case is demonstrated in Fig. 1 for the kinetics $K_{3.3}$. Just below the critical value, $v=0.3332$, we observe a solution of a very large period $T \sim 150$. Each period contains a large excursion piece (a burst of large amplitude and duration) which is followed by an accumulation phase (low amplitude, high frequency, expanding oscillations whose frequency is very close to the linearized frequency $\sqrt{3}$ ). As $v$ drops down, the number of oscillations in the accumulation phase decreases while the shape of the burst is virtually unchanged. For the smallest value of $v$ in Fig. 1 the higher frequency oscillations disappear entirely and we are left with the lower frequency relaxational oscillations consisting of the bursts alone. It appears that, as $v$ decreases, solutions with any finite number of low amplitude oscillations can be observed. Also, numerical evidence suggests that the period of solutions and the number of high frequency oscillations approach infinity as $v$ approaches $v_{\text {cr }}$ from below. Thus, one can naturally conjecture the existence of a saddle-focus type homoclinic orbit with the burst corresponding to the hyperbolic direction and the low amplitude oscillations corresponding to the spiral. As discussed below in Section 5.2, in many respects the scenario depicted in Fig. 1 is very different from the classical Shilnikov case. For this reason we prefer to call it the infinite period bifurcation.

For the other typical scenario (cf. Fig. 3 of [6]), the basic solution loses stability via a Hopf bifurcation and gives rise to harmonic oscillations. As $v$ decreases, one can observe a period doubling cascade leading to chaotic oscillations. With the further decrease in $v$ the solution exits the chaotic regime via an infinite reverse cascade of period doubling that is terminated with a "period 3" solution. As $v$ drops even further, the "period 3" solution undergoes a cascade of period doubling again leading to chaos. As $v$ changes within this chaotic regime, the phase curves deform in such a way that the characteristic "burst corner" (cf. phase diagrams in Fig. 1) becomes well-pronounced. Again the system leaves the chaotic regime through a reverse period-doubling cascade which is terminated with an accumulation-burst pattern containing just one low amplitude oscillation. This, according to our observations, is always the case, that is, if the system enters the region of chaos it exits in one fashion or another for lower values of $v$ to return to simple periodic regimes. Periodic orbits of other finite winding numbers were observed for different values of the kinetic parameter $p$. The reader can find a rather accurate illustration of the scenario just described in Fig. 13 that presents a similar scenario for the ODE approximation.

\section{Finite-dimensional approximations and their spectra}

In the free boundary problem (2.5) and (2.6), it is convenient for our purposes to pass to the moving coordinate system,

$$
z=x-s(t),
$$



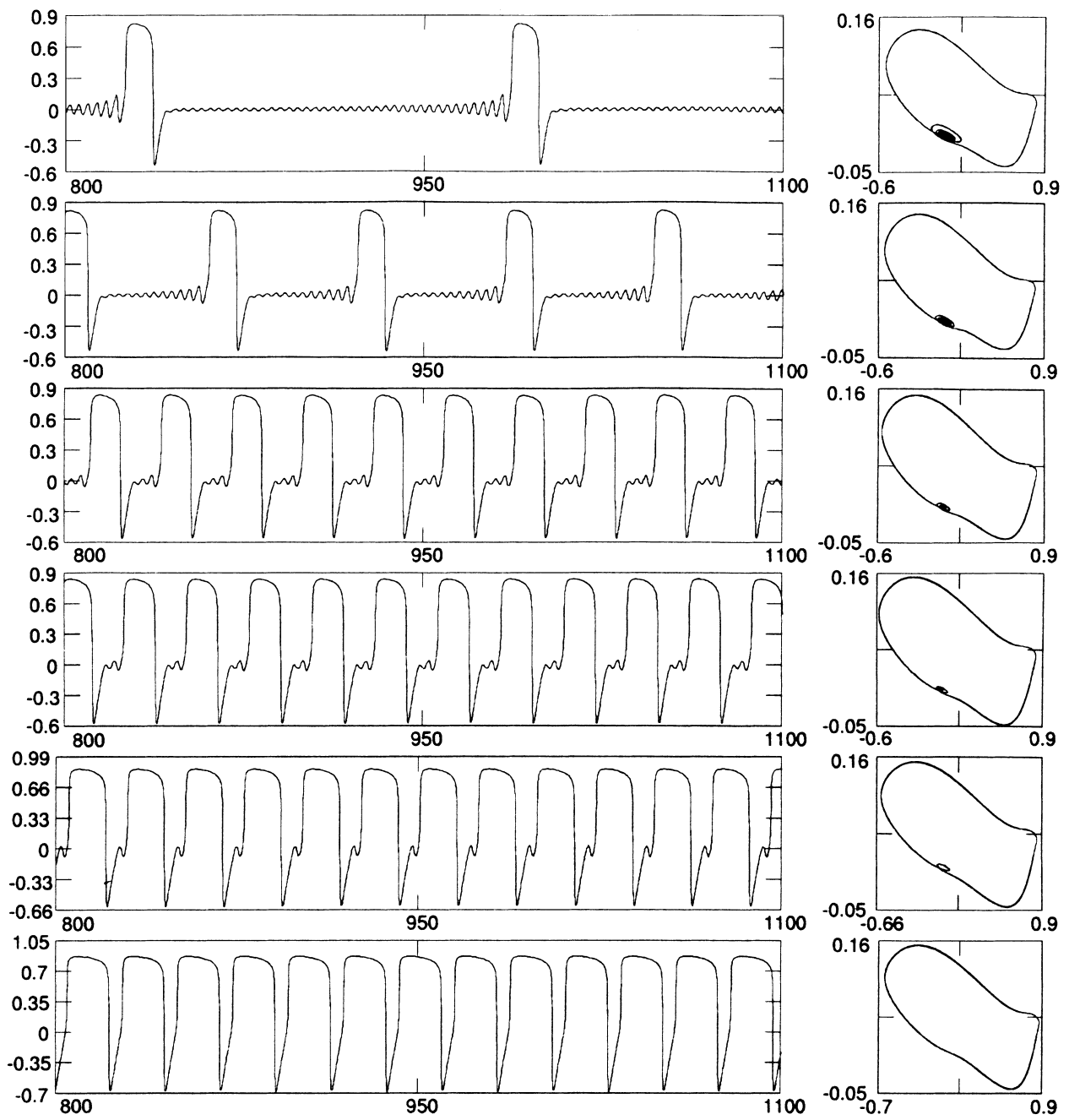

Fig. 1. Infinite period biburcation (the pde case): velocity perturbation profiles, $v(t)$ vs $t$ and projections of the orbits into the plane $\left(v,\left.u\right|_{x=1}\right)$ for $K_{3.3}, v=0.3332,0.331,0.322,0.317,0.3$ and 0.28 .

and to introduce the new dependent variables

$$
w(z, t)=u-u_{b}, \quad v=V+1
$$

which are the deviations from the basic traveling wave solution. In terms of the new variables, the free boundary problem takes the form:

$$
\begin{aligned}
& w_{t}=w_{z z}+(v-1) w_{z}+v u_{b}^{\prime}, \quad z \leq 0, \\
& w(0, t)=v k(v), \quad w_{z}(0, t)=-v, \quad w(-\infty, t)=0,
\end{aligned}
$$

here $k(v)$ is defined as $k(v)=K(1-v)$. Recall that $u_{b}$ is the basic traveling wave which in the moving coordinate frame is given by $u_{b}(z, t)=u_{b}(z)=\mathrm{e}^{z}$, while $u_{b}^{\prime}=\mathrm{e}^{z}$ is its derivative. 
Now we apply the general scheme of pseudo-spectral approximation (see e.g., [16]). As the approximation space $B_{n+1}$ we take the space spanned by the functions of the form $p(z) \mathrm{e}^{z}$, where $p(z)$ is a polynomial of degree $n$. This choice of the approximation space is motivated by the exponential spatial decay of solutions (recall that $z<0$ ). Let

$$
\phi_{k}(z), \quad k=1,2, \ldots, n+1
$$

be a basis in $B_{n+1}$. Then we look for an approximation for the $w$ component of the solution of (4.1) and (4.2) in the form

$$
\tilde{w}(z, t)=\sum_{k=1}^{n+1} a_{k}(t) \phi_{k}(z) .
$$

Next we select $n$ collocation points $z_{1}=0>z_{2}>\cdots>z_{n}$. Possible choices of collocation points will be discussed later, but it should be emphasized that the inclusion of the free boundary point $z=0$ as a collocation point appears to be necessary for obtaining an approximation with correct dynamics. We require $\tilde{w}(z, t)$ and the function $v(t)$ to satisfy (4.1) at the collocation points, and the boundary conditions in (4.2) at 0 . As the result we obtain the following system of $n$ ordinary differential equations and two algebraic equations for the $n+2$ functions $v(t)$ and $\mathbf{a}(t)=\left\{a_{1}(t), a_{2}(t), \ldots, a_{n+1}(t)\right\}^{\mathrm{tr}}$ :

$$
\begin{aligned}
& \Phi \dot{\mathbf{a}}=\Phi D^{2} \mathbf{a}-\Phi D \mathbf{a}+v \Phi D \mathbf{a}+v \Phi \mathbf{b} \\
& \left\langle\mathbf{a}, \boldsymbol{\phi}\left(z_{1}\right)\right\rangle=v k(v), \quad\left\langle\mathbf{a}, D^{\operatorname{tr}} \phi\left(z_{1}\right)\right\rangle=-v .
\end{aligned}
$$

Here $\Phi=\left(\Phi_{i j}\right)$ with $\Phi_{i j}=\phi_{j}\left(z_{i}\right)$ is the $n \times(n+1)$ matrix of the transformation of the $(n+1)$-dimensional approximation space $B_{n+1}$ onto $\mathbf{R}^{n}, \Phi: f \rightarrow\left(f\left(z_{1}\right), f\left(z_{2}\right), \ldots, f\left(z_{n}\right)\right)$. Matrix $D$ is the $(n+1) \times(n+1)$ matrix of differentiation in $B_{n+1}, \mathrm{~d} \Phi / \mathrm{d} z=\Phi D$, finally $\Phi \mathbf{b}$ is the image in $\mathbf{R}^{n}$ of the basic solution $u_{b}(z)=\sum b_{j} \phi_{j}(z)$, and $\boldsymbol{\phi}=\left(\phi_{1}, \ldots, \phi_{n+1}\right)$.

We note that because of linearity in a, changes of basis in $B_{n+1}$ do not affect the system in (4.4) and (4.5), leading just to a rearrangement of equations. From the computational viewpoint, a certain care should be taken in selecting a basis for higher dimensions: orthonormal bases lead to better conditioned matrices.

Even in the case of two differential equations (the space $B_{3}$ ), the pseudo-spectral approximation reveals non-trivial dynamics. In this paper we investigate the case of $B_{4}$ whose dynamics is an amazingly accurate reflection of the behavior of the free boundary problem. A convenient basis in this case is provided by

$$
\phi_{1}(z)=z^{2} \mathrm{e}^{z}, \quad \phi_{2}(z)=z^{3} \mathrm{e}^{z}, \quad \phi_{3}(z)=(1-z) \mathrm{e}^{z}, \quad \phi_{4}(z)=z \mathrm{e}^{z},
$$

(the somewhat unorthodox numeration of polynomials above is dictated by the desire to have the final equations in terms of $a_{1}$ and $a_{2}$ rather than of $\left.a_{3}, a_{4}\right)$. Note that $\phi_{j}\left(z_{1}\right)=0, j=1,2,4 ; \phi_{j}^{\prime}\left(z_{1}\right)=0, j=1,2,3 ; \phi_{3}\left(z_{1}\right)=$ $1 ; \phi_{4}^{\prime}\left(z_{1}\right)=1$. The boundary conditions in (4.5) give the relations

$$
a_{3}=v k(v), \quad a_{4}=-v \text {. }
$$

Upon the substitution of these relations into (4.4), the latter can be resolved with respect to the derivatives to yield the following system of ordinary differential equations:

$$
\begin{aligned}
& \dot{v}=\frac{2 a_{1}-v k-v^{2}}{\nu k^{\prime}}, \\
& \dot{a}_{1}=\alpha\left[-2 a_{1}-3 a_{2}-\frac{2 a_{1}-v k-v^{2}}{2 v k^{\prime}}+\frac{v k}{2}(v+1)+v^{2}-v a_{1}\right]+3 a_{2}(v+1)+v a_{1},
\end{aligned}
$$




$$
\dot{a}_{2}=\beta\left[-2 a_{1}-3 a_{2}-\frac{2 a_{1}-v k-v^{2}}{2 v k^{\prime}}+\frac{v k}{2}(v+1)+v^{2}-v a_{1}\right]+v a_{2},
$$

where prime stands for $\mathrm{d} / \mathrm{d} v$, and $\alpha=-2\left(z_{2}+z_{3}\right) / z_{2} z_{3}, \beta=2 / z_{2} z_{3}$. Everywhere in the next section we use $z_{2}=-1, z_{3}=-2$ which yield the values $\alpha=3, \beta=1$.

We remark that the system in (4.6)-(4.8) takes a particularly simple form (which will not be used in the sequel), if it is written in terms of the new variables:

$$
\xi=2\left(\frac{a_{1}}{\alpha}-\frac{a_{2}}{\beta}\right), \quad \eta=v+\frac{2}{\beta} a_{2}, \quad \zeta=k(v) .
$$

Then it is convenient to treat (4.6)-(4.8) as a system of three differential equations, supplemented by an algebraic relation between $v$ and $\zeta$ :

$$
\begin{aligned}
& \dot{\zeta}=-\zeta+\frac{\alpha}{v}(\xi+\eta-v)-\frac{1}{v} v^{2}, \quad \dot{\xi}=3 \frac{\beta}{\alpha}(\eta-v)(1+v)+v \xi, \\
& \dot{\eta}=(2 \alpha+3 \beta)(v-\eta)-2 \alpha \xi+v(v+1) \zeta+2 v^{2}+v[(1-\alpha)(\eta-v)-\alpha \xi], \zeta=k(v),
\end{aligned}
$$

where the nonlinearities of the differential equations are quadratic. Our preliminary investigation shows that the complicated dynamical behavior discussed in the paper is preserved even if some nonlinearities are dropped.

The system in (4.4) and (4.5) possesses an equilibrium at the origin. The linearized stability of the system about the origin is determined by the following eigenvalue problem:

$$
\lambda \Phi \mathbf{a}=\Phi D^{2} \mathbf{a}-\Phi D \mathbf{a}+v \Phi \mathbf{b}, \quad\left\langle\mathbf{a}, \boldsymbol{\phi}\left(z_{1}\right)\right\rangle=v v,\left\langle\mathbf{a}, D^{\mathrm{tr}} \boldsymbol{\phi}\left(z_{1}\right)\right\rangle=-v .
$$

As in the case $n=3$, the problem can be simplified by selecting a basis for which $\boldsymbol{\phi}\left(z_{1}\right)=(0,0, \ldots, 1,0)$ and $D^{\text {tr }} \boldsymbol{\phi}\left(z_{1}\right)=(0,0, \ldots, 0,1)$. For the $3 \times 3$ system (4.6)-(4.8) with $\alpha=3, \beta=1$, the linear eigenvalue problem can be solved explicitly, and the linearization yields the following eigenvalue equation:

$$
\lambda^{3}+(10-3 / v) \lambda^{2}+(12-3 / v) \lambda+3=0 .
$$

It is easy to see that $\lambda=-1$ is a root for any $\nu$. By factoring this root out, one can see that there are two complex conjugate eigenvalues

$$
\lambda=\frac{1}{2}\left(9-3 / v \pm \sqrt{\left.(9-3 / v)^{2}-12\right)}\right.
$$

crossing into the positive half-plane as $v$ decreases. By coincidence, the threshold value is the same $v_{\mathrm{cr}}=1 / 3$ as for the free boundary problem, with the same pure imaginary eigenvalues $\pm \mathrm{i} \sqrt{3}$. Thus, at $v=1 / 3$ the spectrum of the system is consistent with a Hopf-Shilnikov bifurcation. Symbolic computations (performed with the aid of Maple) demonstrate that the spectra are qualitatively similar for arbitrary choices of collocation points $z_{2}$ and $z_{3}$ : i.e., there is a negative real root and a pair of complex conjugate eigenvalues crossing into the positive half-plane as $v$ decreases. Of course, for arbitrary $z_{2}$ and $z_{3}$, the critical value deviates (rather slightly) from $1 / 3$.

For $n>3$, the linearized eigenvalue problem in (4.11) was solved numerically. In Fig. 2(a) we show eigenvalues of several low dimensional approximations for a fixed $v=0.37$. Note, how the negative eigenvalues tend to "fill" the negative real axis approaching the continuous spectrum of the free boundary problem (cf. Section 3 ). The plots in Fig. 2(b) demonstrate how the pair of complex conjugate eigenvalues crosses the imaginary axis for approximations with different numbers of modes.

As the number of modes in the approximation increases, the placement of collocation points becomes rather important. Our experience shows that a spectrum with the qualitative properties described in the previous paragraph 


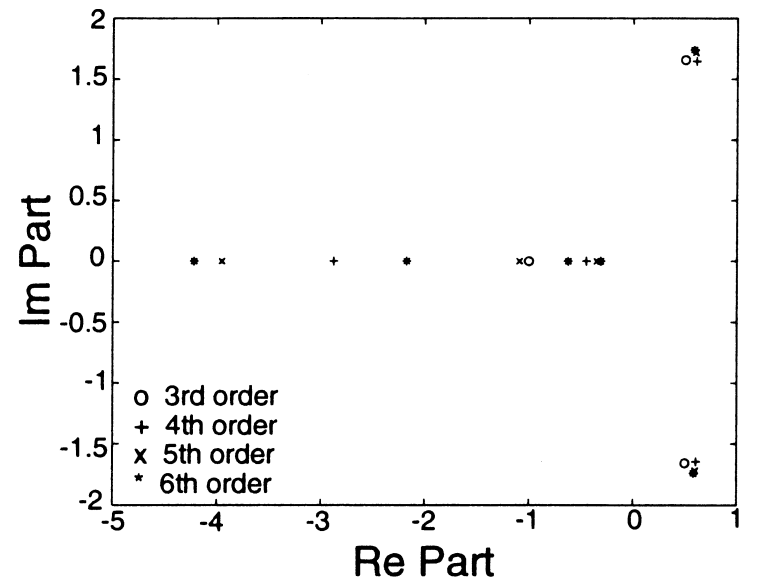

(a)

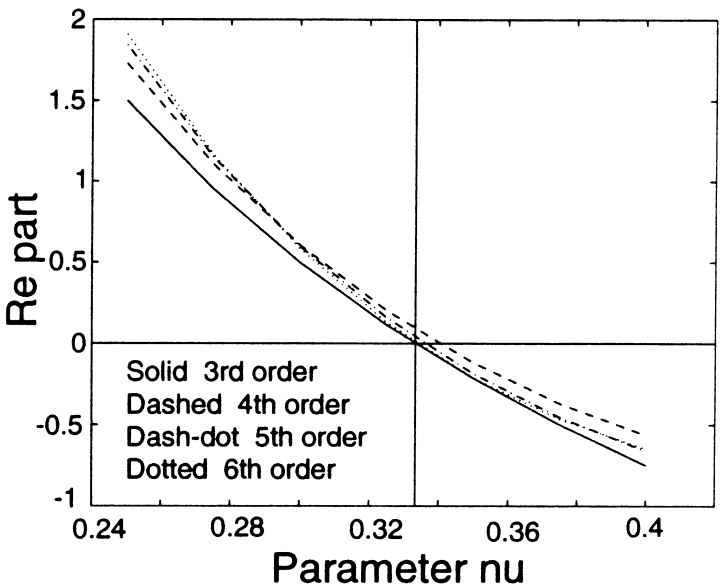

(b)

Fig. 2. (a) Distribution of eigenvalues in the complex plane for approximations with 3, 4, 5 and 6 modes, $v=0.37$; (b) motion of complex roots into the positive half-plane as $v$ decreases.

is attained if collocation points are accumulated near the free boundary $z=0$. Both a simpleminded distribution $z_{k}=z_{k}=-8 k^{2} / 100$ and a bit more sophisticated distribution at zeros of the corresponding Chebyshev polynomial produced spectra which are qualitatively similar, Fig. 3(a). On the other hand, the uniform distribution of collocation points leads to generation of extra pairs of complex roots, Fig. 3(b). However, all these extra roots are very stable, and from the spectral view point, the approximation with uniformly distributed collocation points is not much inferior to the approximation with properly clustered points.

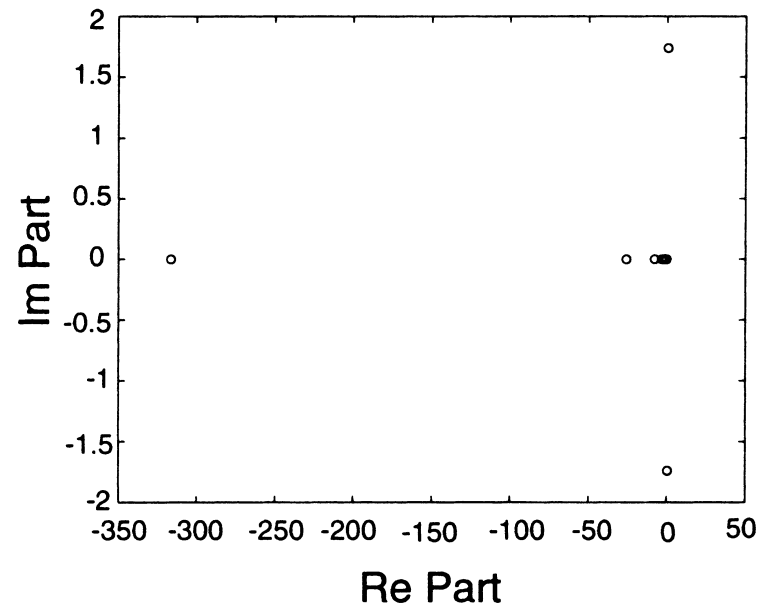

(a)

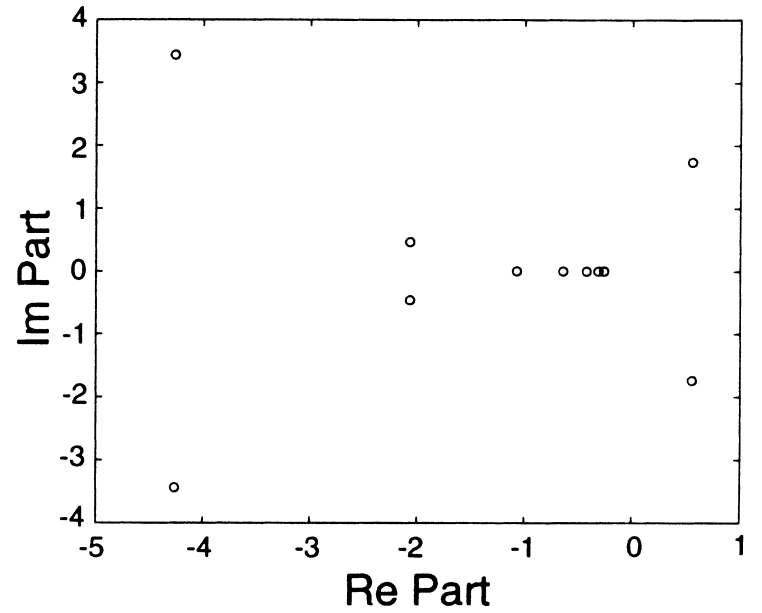

(b)

Fig. 3. Distribution of eigenvalues in the complex plane for $z_{k}=-8 k^{2} / 100$ (a) and $z_{k}=-k$ (b); $n=10 k=0,1,2, \ldots, 10$. 


\section{Dynamics of the three-mode approximation}

In this section we describe and classify fascinating dynamical scenarios that are exhibited by the $3 \times 3$ system of ODEs (4.6)-(4.8). Dynamical scenarios of this section are strikingly similar to the dynamical scenarios for the free boundary model that have been discussed in Section 3.

All the time histories and phase portraits presented below are obtained through direct numerical simulations with initial conditions close to the equilibrium at 0 . Thus, periodic time histories are in fact time evolutions which are attracted by stable periodic solutions; as a rule the attraction in these cases is extremely strong. For convenience of comparison with the free boundary problem, we use here the same phase plane projection into the $\left.v u\right|_{x=-1}$ plane as in Section 3. It is easily seen that $\left.u\right|_{x=-1}=\left(a_{1}-a_{2}\right) / e$. We note that the direct numerical simulations of this section were repeated for a four-mode pseudo-spectral approximation of the original free boundary problem. Simulations on the $4 \times 4$ system of ODEs gave a virtually identical reproduction of time histories and phase portraits observed for the $3 \times 3$ case.

All the bifurcation diagrams of this section represent the $L_{2}$-norm of solutions against a bifurcation parameter (which is almost always $v$ ), where $L_{2}$-norm is defined as normalized by the period,

$$
\lim _{T \rightarrow \infty} \frac{1}{\sqrt{T}}\left[\int_{0}^{T}\left(v^{2}(t)+a_{1}(t)^{2}+a_{2}(t)^{2}\right) \mathrm{d} t\right]^{1 / 2}
$$

Recall that $v, a_{1}, a_{2}$ are components of a solution. This norm is a standard norm for almost periodic functions. For periodic functions it produces the $L_{2}$-norm normalized by the period. The bifurcation diagrams are generated by the continuation software AUTO94 [4], which computes both dynamically stable and dynamically unstable periodic solutions.

Recall from Section 4, that when $v$ drops below the critical level $v_{\mathrm{cr}}=1 / 3$, two complex conjugate eigenvalues of the linearized system cross the imaginary axis into the right half-plane. The crossing is transversal, and the basic solution loses stability via a Hopf bifurcation. The character of the bifurcation and stability properties of bifurcating periodic solutions depend on the structure of nonlinearity, which in our case is governed by the parameter $p$ (as before, we consistently use the nonlinear kinetic function from (3.5)). Numerical computation of Floquet exponents of bifurcating solutions was performed with the aid of AUTO94. The computations show that there are two critical values of $p, p_{1}=0.55$ and $p_{2}=2.37$, at which the bifurcation switches from a subcritical to a supercritical case. Typical bifurcation diagrams for $p=0.4,1.5,3$ are presented in Fig. 4. For both subcritical diagrams, Fig. 4(a,c), the solutions corresponding to the points of the bifurcation branch for small $v-v_{\mathrm{cr}}$ are dynamically unstable and cannot be observed in simulations. Meanwhile, dynamics that do occur for $p<p_{1}$ and $p>p_{2}$ differ dramatically.

\subsection{Subcritical bifurcation: "hard” loss of stability}

The $p<p_{1}$ regimes exhibit a classical "hard" exchange of stability. As $v$ drops below $v_{\mathrm{cr}}$ the zero solution loses stability giving rise to a limit cycle of finite magnitude. On the bifurcation diagram, the bifurcation corresponds to a jump upwards from 0 to the stable part of the bifurcation branch. In Fig. 5 we present a few time histories and phase portraits for decreasing $v$.

Even very close to the threshold of stability, the periodic solution is of order one amplitude. In contrast with harmonic oscillations, the periodic solutions are characterized by the prolonged plateaus separated by the zones of a very steep change (in the context of nonlinear oscillations, such oscillations are sometimes called relaxational). As $v$ decreases the amplitude and period increase gradually. 

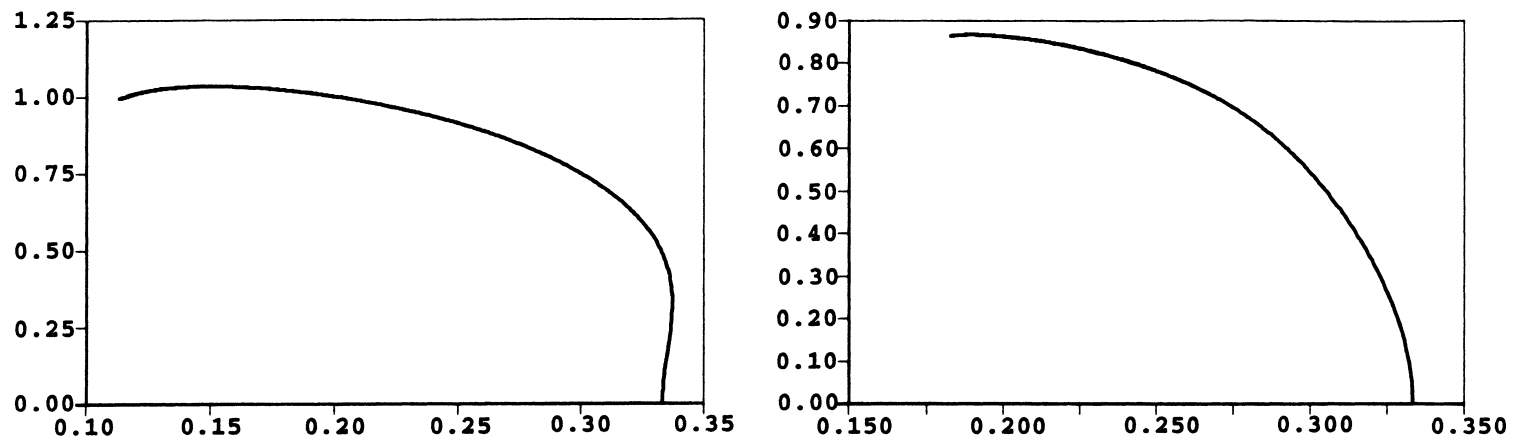

(a)

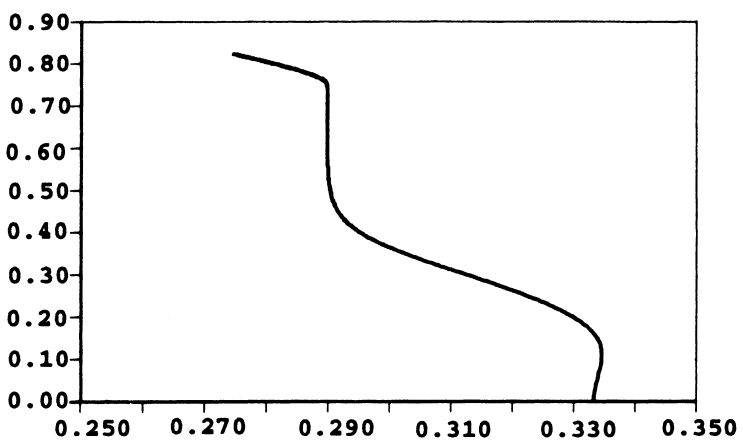

(b)

(c)

Fig. 4. Typical bifurcation branches, $L_{2}$-norm of periodic solutions vs $v$ : (a) subcritical $p=0.4<p_{1}$; (b) supercritical $p_{1}<p=1.5<p_{2}$; (c) subcritical $p>p_{2}$.

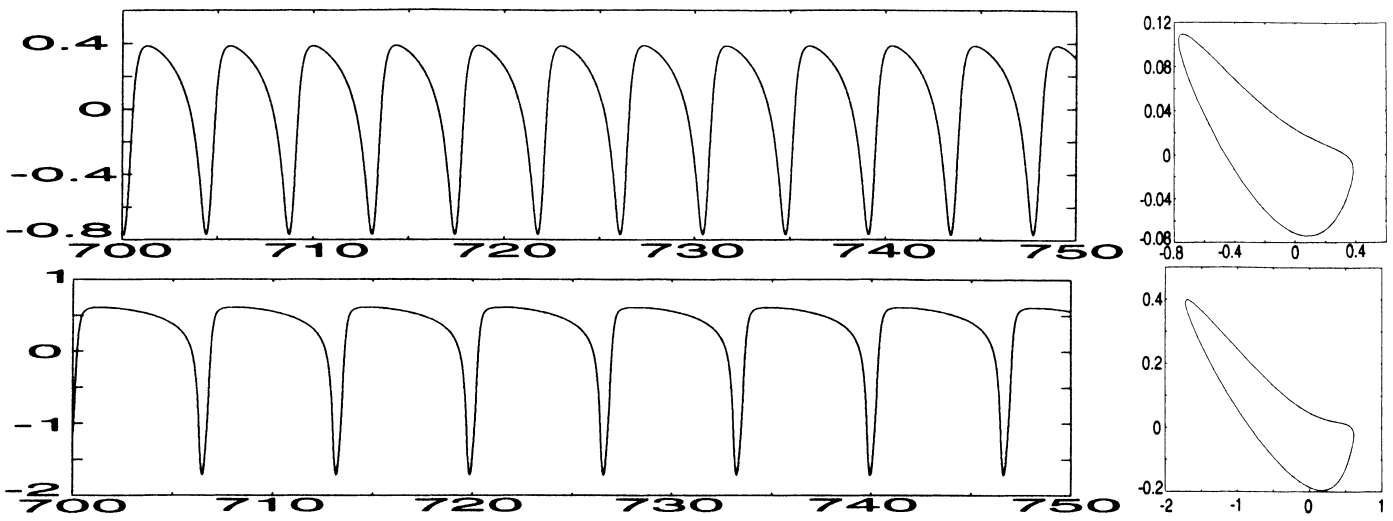

Fig. 5. Hard loss of stability, $p=0.4$ : velocity perturbation profiles, $v(t)$ vs $t$ and projections of the orbits into the plane $\left(v,\left.u\right|_{x=1}\right)$ for $v=0.3333$, and 0.3 .

\subsection{Subcritical bifurcation: an infinite period bifurcation}

The $p>p_{2}$ regimes demonstrate the infinite period bifurcation that appears identical to its counterpart for the free boundary problem. The sequence depicted in Fig. 6 should be compared to the one in Fig. 1. Again we see 

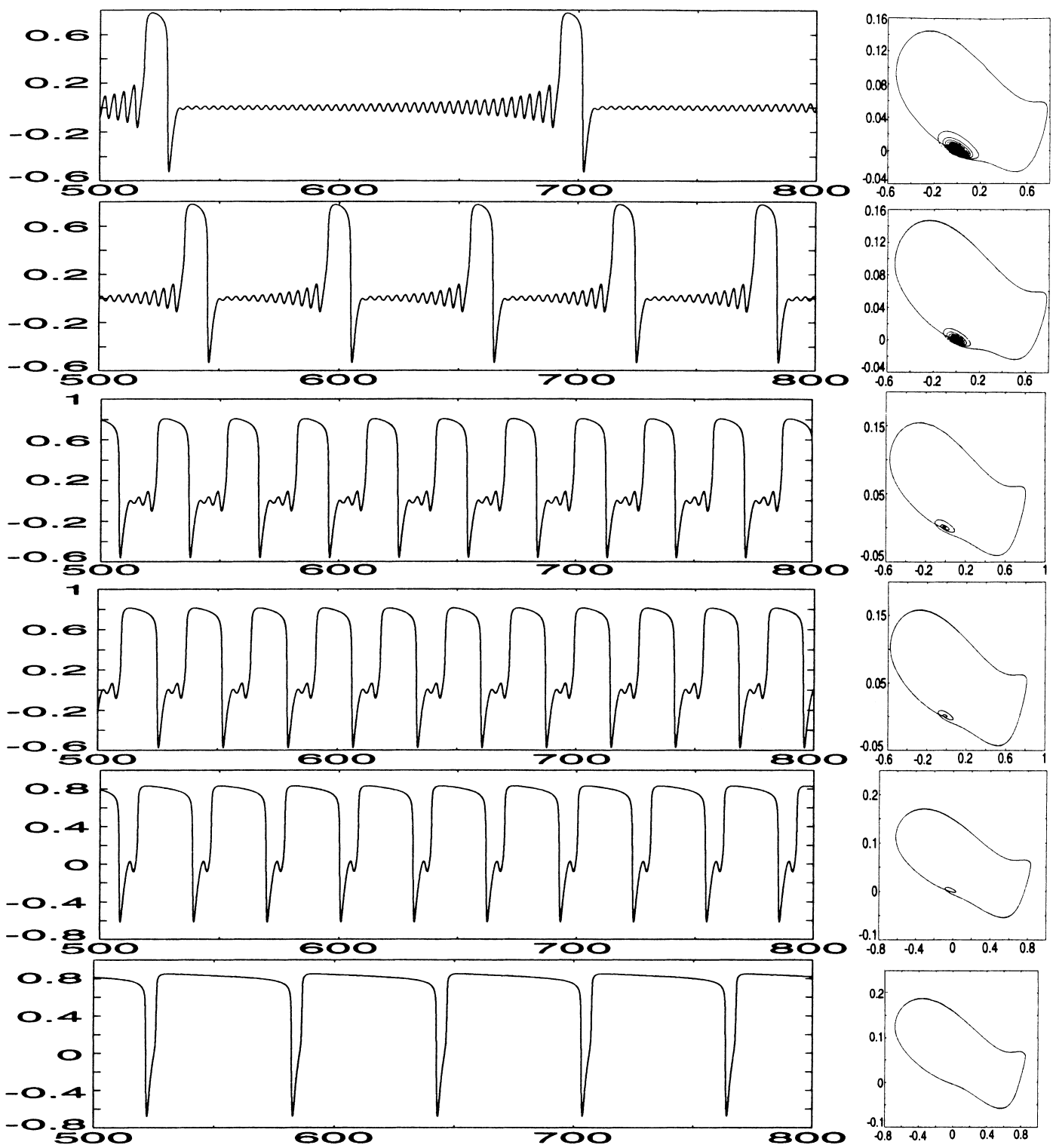

Fig. 6. The infinite period bifurcation case: velocity perturbation profiles $v(t)$ vs $t$ and projections of the orbits into the plane $\left(v,\left.u\right|_{x=1}\right)$ for $p=3, v=0.3325,0.33,0.32,0.315,0.3$ and 0.28 .

a series of higher frequency oscillations whose period is close to the linear period (i.e., the period corresponding to the pure imaginary eigenvalues of the linearized problem). The higher frequency oscillations are followed by a prolonged larger amplitude burst. The total period of the solution approaches infinity as $v$ approaches $v_{\mathrm{cr}}$ (for the first plot in Fig. 6, $T \sim 200$ for $v=0.3325$ ). This structure of solutions, and the nature of the linearized spectrum at $v_{\mathrm{cr}}$ (two complex-conjugate eigenvalues crossing into the positive complex half-plane, and one negative order one eigenvalue) seem to suggest the existence of a homoclinic orbit and a Hopf-Shilnikov type bifurcation. We will demonstrate that the situation is dramatically different from the classical Shilnikov case $[3,14,17]$. 


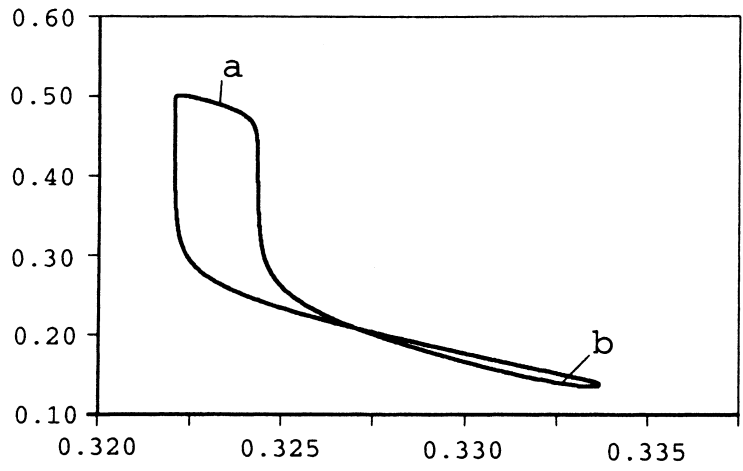

(a)

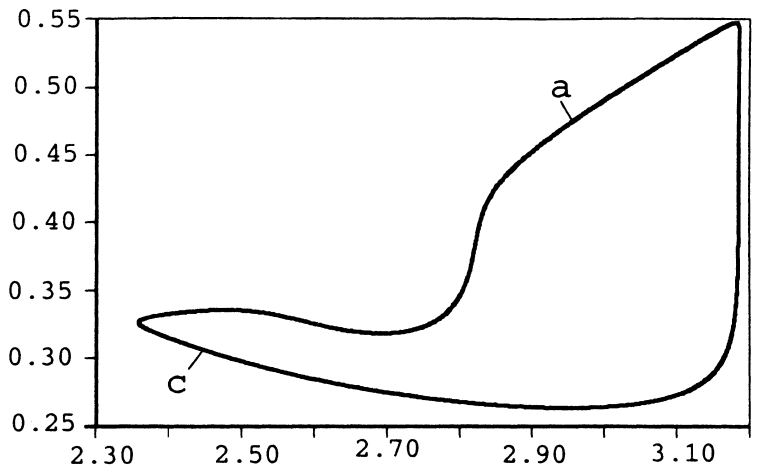

(b)

Fig. 7. Bifurcation curves for periodic solutions with four low-amplitude oscillations: (a) continuation in $v$, the $L_{2}$-norm vs $v, p=3$; (b) continuation in $p$, the $L_{2}$-norm vs $p, v=0.324$.

As was discussed above, for small $v-v_{\mathrm{cr}}$ the piece of the bifurcation branch in Fig. 4(c) which is adjacent to the $v$-axis corresponds to dynamically unstable solutions. Clearly, the continuation from the basic solution does not allow us to connect to dynamically stable solutions of Fig. 6. To investigate bifurcations of dynamically stable solutions, we started the continuation process from such a solution which was obtained as a result of direct numerical simulation. In contrast with the classical Shilnikov bifurcation, instead of an oscillatory bifurcation curve, with the magnitude of oscillations gradually decaying (a "snake") along which the period is continually increasing, the continuation produced a closed branch of solutions of roughly the same period.

A typical example is depicted in Fig. 7(a) (continuation in $v$ for fixed $p$ ) and in Fig. 7(b) (continuation in $p$ for fixed $v$ ). All the periodic solutions on the closed continuation branches have the same number (five for this example) of oscillations, whose shape is being deformed along the way (see Fig. 8 for typical time evolutions of $v$ ). Only small vicinities of the points marked "a" in Fig. 7 correspond to stable periodic solutions as determined by AUTO94 (we remind that to determine stability, the code computes the corresponding Floquet exponents). It is worth mentioning that both bifurcation curves are topological circles, and therefore in the $\|\cdot\|_{2}-p-v$ bifurcation space, the surface of periodic solutions with the same number of oscillations is a topological torus.

In Fig. 9 we present a sequence of closed curves corresponding to consecutive numbers of oscillations. In contrast with the classical Shilnikov bifurcation, in this scenario there is no continuous transition in "adding" high frequency oscillations. To avoid cluttering the figure we present consecutive closed curves only for 2, 3, 4, 5 low amplitude oscillations and then for 14. As the number of low amplitude oscillations in the periodic solution increases, the corresponding figure eight curves become smaller and smaller and converge to the point $(1 / 3,0)$. For higher number of high frequency oscillations, only selected dynamically stable solutions are shown in Fig. 9; the dots correspond to $8,12,16,19,23$, and 90 accumulation oscillations.

The top parts of figure-eight curves correspond to dynamically stable solutions, thus the envelope of these figure-eight curves is the curve along which stable periodic solutions bifurcate. The envelope curve approaches the point $(1 / 3,0)$ along a parabola. In this sense the infinite period bifurcation is similar to the standard Hopf bifurcation, for which the norm of bifurcating solutions is also on the order of $\sqrt{v_{\mathrm{cr}}-v}$. Of course, unlike the standard Hopf bifurcation case, only the frequency of small oscillations is related to the linear period, while the overall period is infinite.

We note that in numerical simulations, stable periodic solutions attract other solutions at different rates. For the values of $v$ close to the transition between $n$ and $n+1$ low amplitude oscillations, the rate of attraction is substantially slower than for the values of $v$ away from the transition level. Close to the transition level, before 
(a)

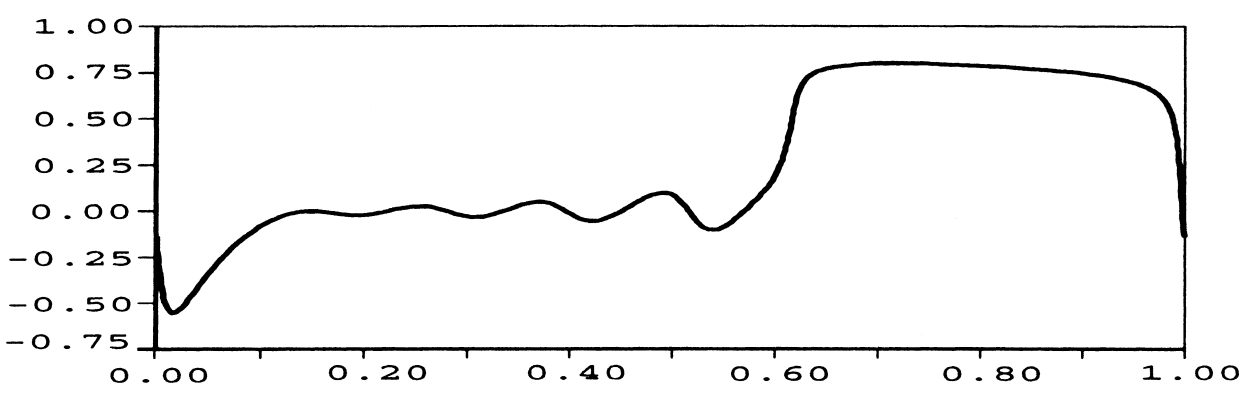

(b)

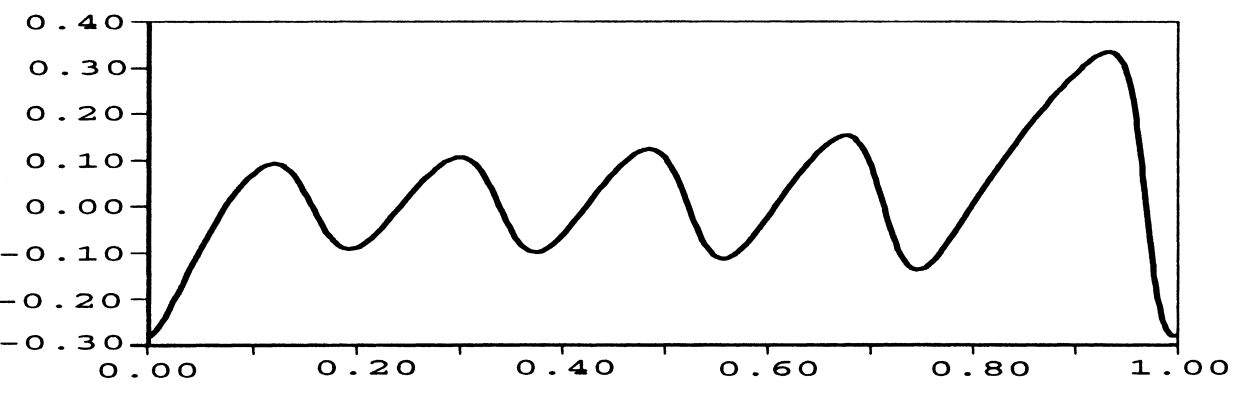

(c)

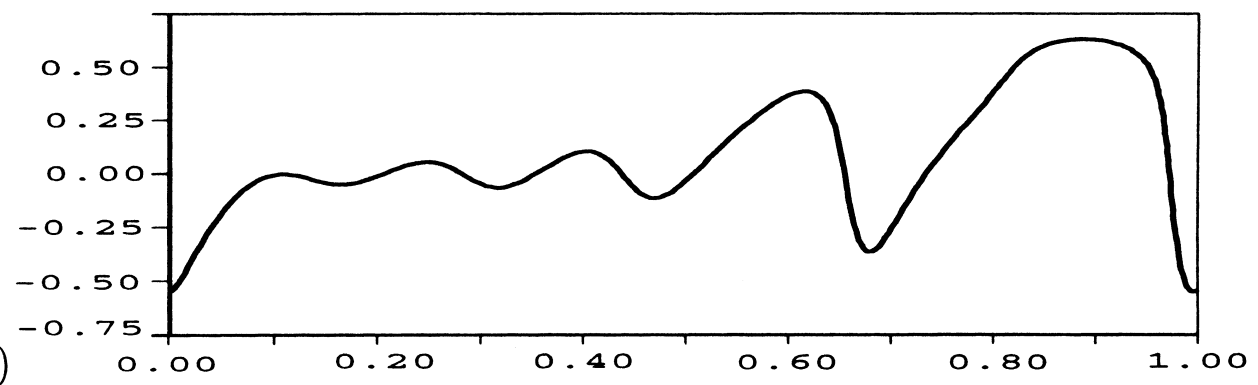

Fig. 8. Typical time histories $v(t)$ vs $t$ for periodic solutions with four low-amplitude oscillations. The time histories correspond to the points marked "a", "b", and "c" in the previous figure.

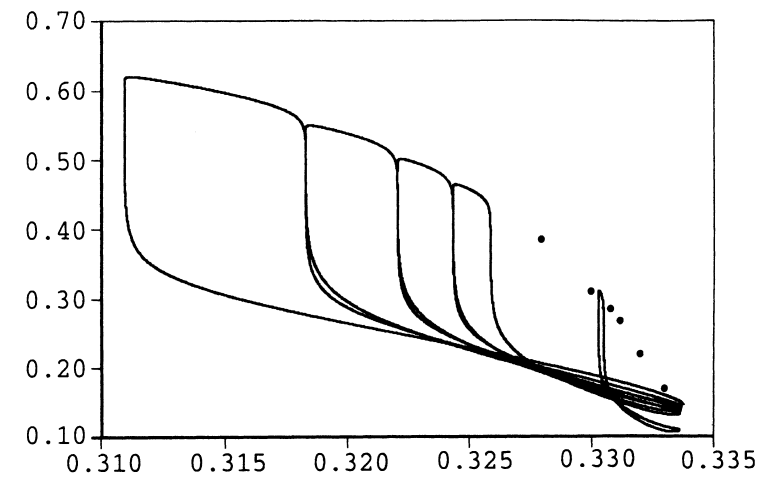

Fig. 9. A series of bifurcation curves, the $L_{2}$ norm of periodic solutions for solutions with increasing number of oscillations, $p=3$. 




Fig. 10. A simple transition from harmonic oscillations to relaxation oscillations, velocity perturbation profiles, $v(t)$ vs $t$, and projections of the orbits into plane $\left(v,\left.u\right|_{x=1}\right), p=1.5, v=0.33,0.315$, and 0.29

settling to a periodic trajectory with $n$ small oscillations, the numerical solution "hesitate" and may pick up, at random, different numbers of small oscillations separated by large bursts.

\subsection{Supercritical bifurcation $\left(p_{1}<p<p_{2}\right)$}

In the weakly nonlinear regime, the periodic solutions that bifurcate from the trivial solution are harmonic oscillations with the period close to the linear period and the amplitude on the order of $\sqrt{v_{\mathrm{cr}}-v}$, as prescribed by the bifurcation diagram Fig. 4(b). As $v$ moves into more nonlinear regions however, there is a variety of possible dynamical scenarios, depending on the value of the kinetic parameter $p$.

For the regimes with $p<p_{3}=1.92$ dynamics are qualitatively similar. They are illustrated by the sequence in Fig. 10. As $v$ decreases, harmonic oscillations gradually become more and more relaxational while their period is gradually increasing.

At $p \simeq 1.92$ a new qualitative feature appears which is illustrated in the sequence in Fig. 11. At $v=0.323$ the harmonic periodic solution experiences period doubling. As $v$ continues to drop, the larger oval of the phase portrait develops a characteristic "corner" that corresponds to the burst phase of the infinite period bifurcation sequence. Finally, through the reverse period doubling, a simple periodic solution is created, it contains the periodically repeated burst-like phase alone. As $p$ increases, this scenario evolves in the following fashion (Fig. 12) : a harmonic oscillation undergoes two period doublings, then the ovals form corners, and through the cascade of two reverse doublings a single burst solution is born. As $p$ increases further, the scenario of cascade-deformation-reverse cascade was observed with 3 and 4 period doublings. Apparently, cascades with any $2^{k}$ number of ovals should exist.

Yet another interesting qualitative feature appears as $p$ increases beyond $p=2.1$. First, as $v$ decreases, a periodic solution undergoes an infinite series of period doubling and becomes chaotic. With the further decrease in $v$ the solution exits the chaotic regime via an infinite reverse cascade of period doubling that is terminated with a "period 3" solution. As $v$ drops even further, the "period 3" solution undergoes a cascade of period doubling leading to 


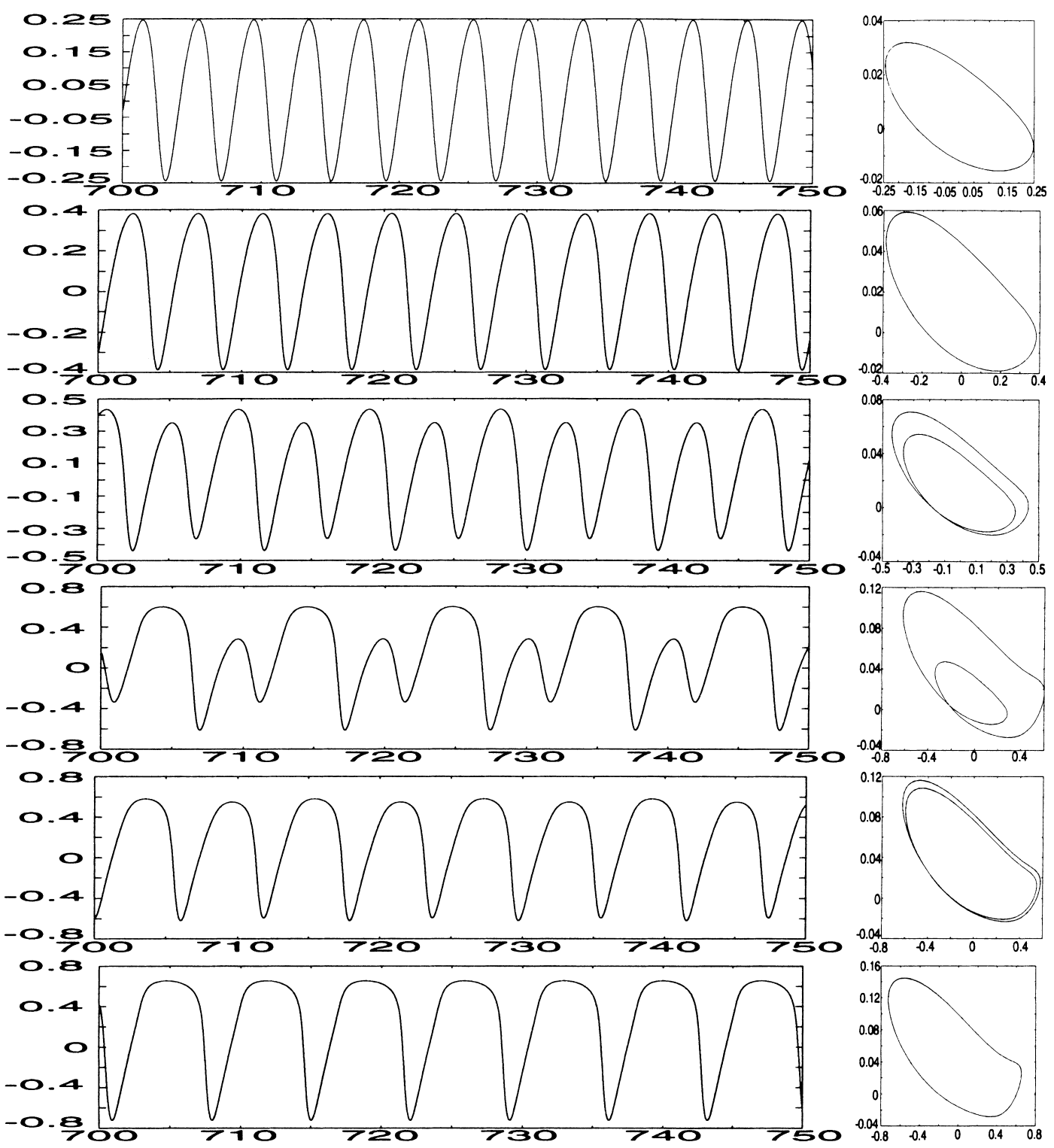

Fig. 11. A transition from harmonic oscillations to burst like oscillations through direct and reverse period doubling, velocity perturbation profiles, $v(t)$ vs $t$ and projections of the orbits into the plane $\left(v,\left.u\right|_{x=1}\right) ; p=2.0, v=0.33,0.324,0.323,0.315,0.3101$, and 0.3 .

chaos. As $v$ changes within this chaotic regime, the phase curves deform in such a way that the characteristic "burst corner" becomes well-pronounced. Again the system leaves the chaotic regime through a reverse period-doubling cascade which is terminated with an accumulation-burst pattern. Depending on the value of parameter $p$, we observed patterns with 2, 3, 4, and 5 accumulation oscillations; apparently any number of these oscillations can be obtained. Finally, as $v$ moves even further into the nonlinear region, the number of accumulation oscillations is consecutively reduced to two through the mechanism described in Section 5.2. After that the last accumulation oscillation disappears through a reverse period doubling, and a pure burst periodic solution arises. The scenario just 

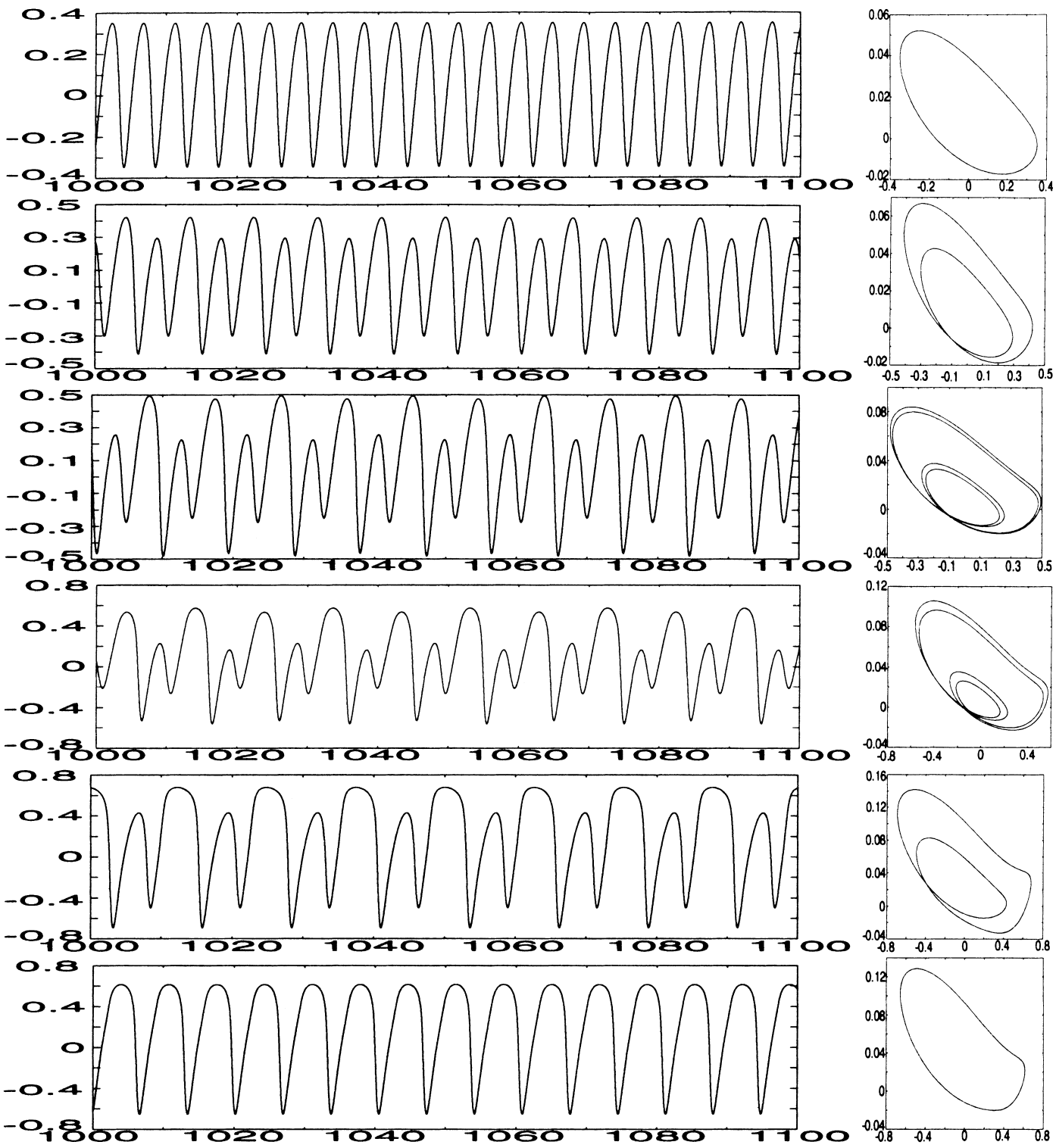

Fig. 12. A transition from harmonic oscillations to burst like oscillations through a cascade of direct and reverse period doubling, velocity perturbation profiles, $v(t)$ vs $t$, and projections of the orbits into the plane $\left(v,\left.u\right|_{x=1}\right), p=2.08, v=0.326,0.325,0.323,0.3195,0.3063$, and 0.306

described is illustrated in Fig. 13. The second reverse cascade there ends with the burst-accumulation pattern which contains three low amplitude oscillations.

\section{Conclusion}

We have presented a three-dimensional system of ordinary differential equations that reveals very rich dynamics and novel mechanisms of exchange of stability. In particular it demonstrates a critical and subcritical Hopf bifurcation, multiple cascades of period doubling, and a novel type of infinite period bifurcation. 


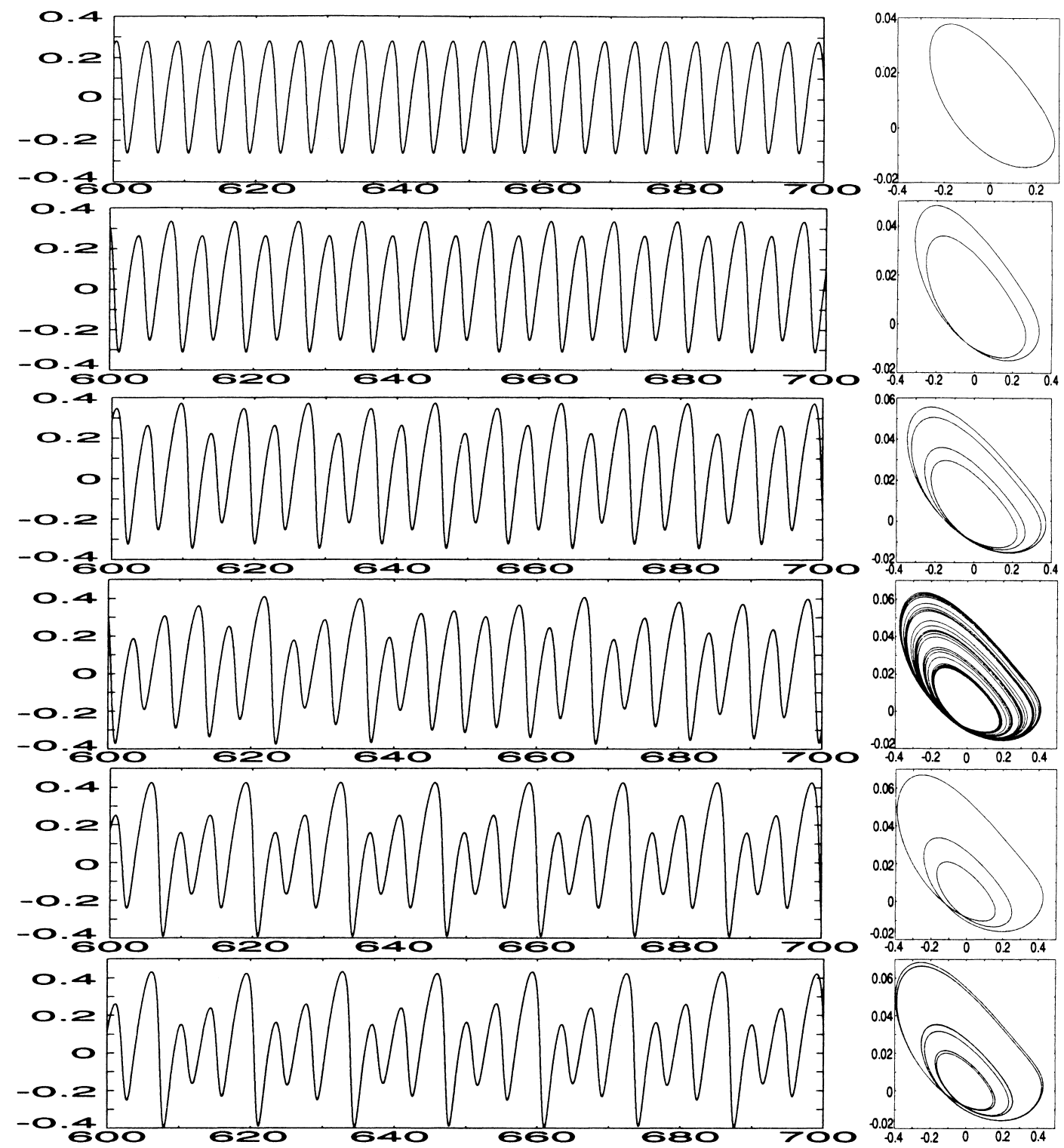

Fig. 13. A transition from harmonic oscillations to burst like oscillations through a cascade of direct and reverse period doubling, period 3 , and another direct-reverse period doubling cascade, velocity perturbation profiles, $v(t)$ vs $t$, and projections of the orbits into the plane $\left(v,\left.u\right|_{x=1}\right), p=2.3, v=0.33,0.329,0.3285,0.3281,0.32805,0.328,0.327,0.3245,0.3235,0.322,0.315,0.31$, and 0.29 .

The system has clear physical origins and approximates dynamics of the underlying infinitely dimensional (pde) problem extremely accurately. This gives a strong indication that the free boundary problem itself possesses a finite-dimensional attractor. We address this issue in a work in progress. We prove that a version of the free boundary problem on a finite spatial interval does possess a finite-dimensional attractor. Moreover, the dimension does not depend on the length of the interval. These results will appear in the nearest future (see [13]).

We believe the three-dimensional system of ordinary differential equations of the present paper is a very interesting example of a relatively simple low-dimensional dynamical system that preserves important features of its physically 

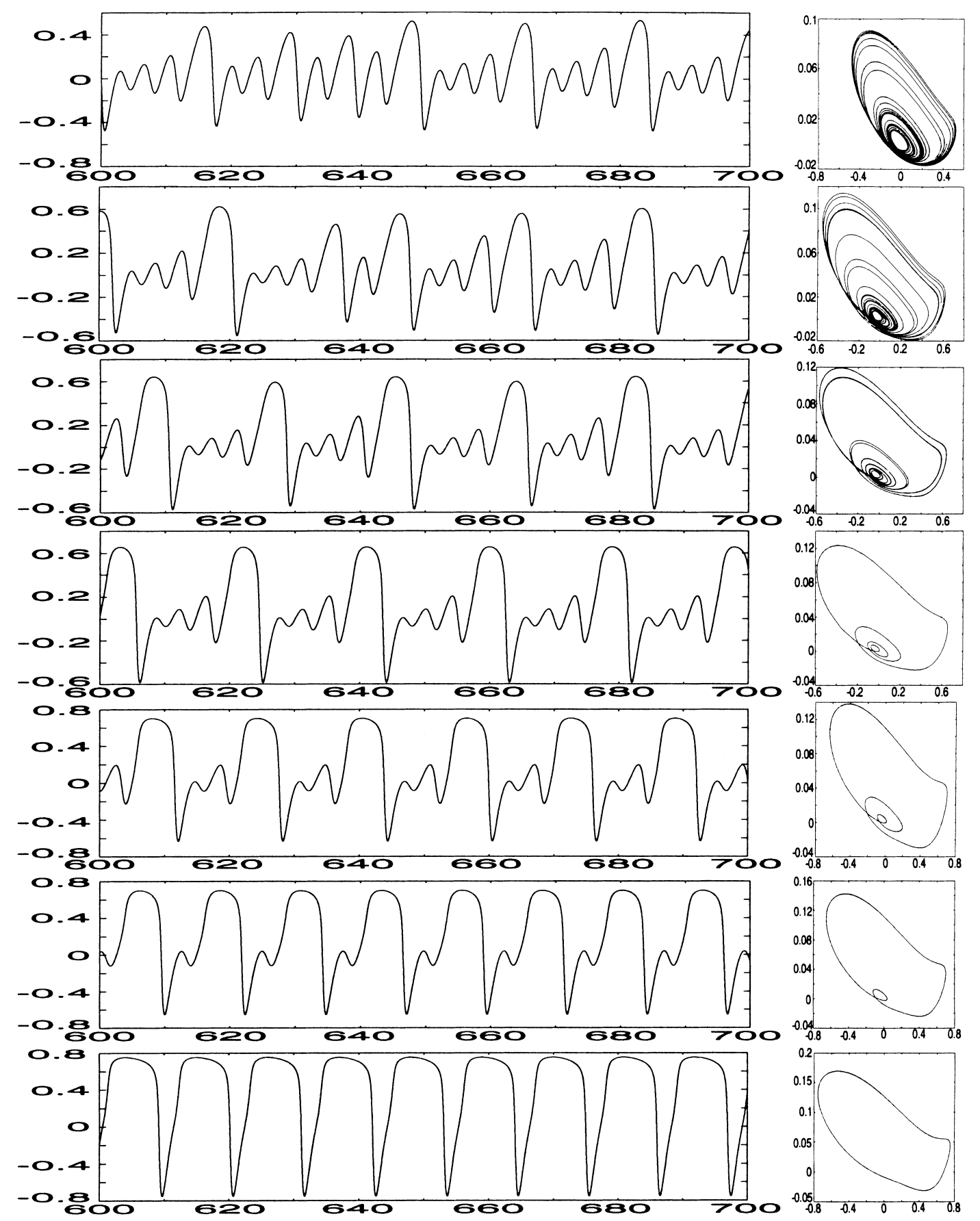

Fig. 13. (Continued). 
motivated and dynamically rich PDE source, even in a variety of strongly nonlinear regimes. It appears that not all the nonlinearities of the system are of equal importance, and that some of them can be dropped without loosing any dynamical scenarios. It would be very interesting to find a normal form for the system that would allow to clarify the origin and structure of the unusual dynamics we have discussed in the paper.

\section{Acknowledgements}

Michael Frankel would like to acknowledge support in part by NSF through grant DMS-9623006. Gregor Kovačič and Ilya Timofeyev would like to acknowledge support in part from the US Department of Energy through grant DE-FG02-93ER25154, the National Science Foundation through grants DMS-9502142 and DMS-9510728, and the Alfred P. Sloan Foundation through a Sloan Research Fellowship. Victor Roytburd would like to acknowledge support in part by NSF through grant DMS-9704325. The authors are grateful to the referee for several helpful suggestions.

\section{References}

[1] I. Brailovsky, G. Sivashinsky, Chaotic dynamics in solid fuel combustion, Physica D 65 (1993) 191-198.

[2] A. Bayliss, B. Matkowsky, M. Minkoff, Period doubling gained, period doubling lost, SIAM J. Appl. Math. 49 (1989) 1047-1063.

[3] M. Bosch, C. Simó, Attractors in a Shilnikov-Hopf scenario and a related one-dimensional map, Physica D 62 (1993) $217-229$.

[4] E. Doedel, X. Wang, T. Fairgrieve, AUT094, Software for continuation and bifurcation problems, Applied Mathematics Report, California Institute of Technology, 1994.

[5] M.L. Frankel, On the nonlinear evolution of a solid-liquid interface, Phys. Lett. A 128 (1988) 57-60.

[6] M.L. Frankel, V. Roytburd, Dynamical portrait of a model of thermal instability: cascades, chaos, reversed cascades and infinite period bifurcations, Int. J. Bifur. Chaos 4 (1994) 579-593.

[7] M.L. Frankel, V. Roytburd, G. Sivashinsky, A sequence of period doubling and chaotic pulsations in a free boundary problem modeling thermal instabilities, SIAM J. Appl. Math. 54 (1994) 1357-1374.

[8] M.L. Frankel, V. Roytburd, A free boundary problem modeling thermal instabilities: well-posedness, SIAM J. Math. Anal. 25 (1994) $1357-1374$.

[9] M.L. Frankel, V. Roytburd, A free boundary problem modeling thermal instabilities: stability and bifurcation, J. Dynamics Differential Equations 6 (1994) 447-486.

[10] M.L. Frankel, V. Roytburd, On a free boundary problem related to solid-state combustion, Comm. Appl. Nonlinear Anal. 2 (1995) 1-22.

[11] M.L. Frankel, V. Roytburd, Finite-dimensional model of thermal instability, Appl. Math. Lett. 8 (1995) 39-44.

[12] M. Frankel, M. Qu, V. Roytburd, On a free interface problem modeling solid combustion and rapid solidification in infinite medium, in: Dynamical Systems and Applications, World Scientific series in Applicable Analysis, vol. 4, 1995, pp. 263-278.

[13] M.L. Frankel, V. Roytburd, Finite-dimensional attractors for a free boundary model of a thermal instability, in preparation.

[14] P. Glendinning, C. Sparrow, Local and global behavior near homoclinic orbits, J. Stat. Phys. 35 (1984) 645-696.

[15] S.M. Gol'berg, M.I. Tribelskii, On laser induced evaporation of nonlinear absorbing media, Zh. Tekh. Fiz. (Sov. Phys.-J. Tech. Phys.) 55 (1985) 848-857 (in Russian).

[16] D. Gottlieb, S. Orszag, Numerical Analysis of Spectral Methods: Theory and Applications, SIAM, Philadelphia, PA, 1977.

[17] P. Hirschberg, E. Knobloch, Shilnikov-Hopf bifurcation, Physica D 62 (1993) 202-216.

[18] J.S. Langer, Lectures in the theory of pattern formation, in: J. Souletie, J. Vannimenus, R. Stora (Eds.), Chance and Matter, Elsevier, Amsterdam, 1987.

[19] B.J. Matkowsky, G.I. Sivashinsky, An asymptotic derivation of two models in flame theory associated with the constant density approximation, SIAM J. Appl. Math 37 (1979) 686-689.

[20] A.G. Merzhanov, A.K. Filonenko, I.P. Borovinskaya, New Phenomena in Combustion of Condensed Systems, Dokl. Akad. Nauk USSR, 208 (1973) 892-894 (Soviet Phys. Dokl. 208 (1973) 122-125).

[21] A.G. Merzhanov, SHS processes: combustion theory and practice, Arch. Combustionis, 1 (1981) 23-48.

[22] K.G. Shkadinsky, B.I. Khaikin, A.G. Merzhanov, Propagation of a Pulsating Exothermic Reaction Front in the Condensed Phase, Combust. Expl. Shock Waves 7 (1971) 15-22.

[23] W. Van Saarloos, J. Weeks, Surface undulations in explosive crystallization: a nonlinear analysis of a thermal instability, Physica D 12 (1984) 279-294.

[24] A. Visintin, Stefan problem with a kinetic condition at the free boundary, Annali di Matematica Pura ed Applicata 146 (1987) 97-122.

[25] Ia.B. Zeldovich, G.I. Barenblatt, V.B. Librovich, G.M. Makhviladze, The Mathematical Theory of Combustion and Explosions, Consultants Bureau, Washington, DC, 1985. 